\title{
Too Small to Form a Galaxy: How the UV Background Determines the Baryon Fraction
}

\author{
M. Hoeft ${ }^{1}$, G. Yepes ${ }^{2}$ and S. Gottlöber ${ }^{3}$ \\ ${ }^{1}$ Jacobs University Bremen, Germany \\ m.hoeft@jacobs-university.de \\ ${ }^{2}$ Universidad Autonoma de Madrid, Spain \\ ${ }^{3}$ Astrophysikalisches Institut Potsdam, Germany
}

\begin{abstract}
The cosmic ultraviolet background (UVB) heats the intergalactic medium (IGM), as a result the gas in dark matter halos below a certain mass is too hot to cool within a Hubble time. The UVB effectively suppresses the formation of dwarf galaxies. Using high resolution cosmological hydrodynamical simulations we show that photo heating leads to small baryon fractions in halos below $\sim 6 \times 10^{9} h^{-1} M_{\odot}$, independent of the cosmic environment. The simulations are carried out assuming a homogeneous UVB with flux densities as given by Haardt \& Madau (1996). A halo may stop to condense gas significantly after the universe is reionised, namely when its mass falls below the characteristic mass scale set by the photo heating. Assuming a spherical halo model we derive this characteristic mass analytically and identify the main mechanisms that prevent the gas from cooling in small halos. The theoretically derived characteristic mass is smaller than the one obtained from observations. Increasing the energy per ionising photon by a factor between four and eight would be sufficient to reconcile both. This is equivalent to an average temperature of the IGM of $\sim 10^{4} \mathrm{~K}$. In this sense the faint end of the luminosity function may serve as a calorimeter for the IGM.
\end{abstract}

Keywords. galaxies: formation, galaxies: dwarf, ultraviolet: galaxies, methods: numerical

\section{Introduction}

The faint end of the luminosity function of galaxies indicates that either only a small fraction of dark matter halos with masses below a few times $10^{10} h^{-1} M_{\odot}$ ('dwarf halos') contain galaxies, or that galaxies in dwarf halos show on average a high mass-to-light ratio (provided that the cold dark matter scenario is a good description of our universe). It has been argued decades ago that the IGM is highly ionised by a cosmic UVB. The Ly $\alpha$ forest proves excellently that only a tiny fraction of hydrogen is neutral. The energy put into the IGM in each photoionisation event leads to temperatures about $\sim 10^{4} \mathrm{~K}$ at present. We have shown in a preceding work (Hoeft et al., 2006) that assuming the canonical model of the UVB (Haardt \& Madau, 1996) the resulting characteristic mass of dark matter halos with baryon deficiency amounts to $6 \times 10^{9} h^{-1} M_{\odot}$. It is too small to turn a sufficient number of dwarf halos dark.

Here, we will show in detail how photo heating hampers the gas condensation in dwarf halos. To this end we present results from simulations including cooling and photo heating and set up an analytical model. We argue that the lower end of the galaxy luminosity function is essentially a measure for the heat input into the IGM.

\section{Simulations}

Our simulations have been run with the parallel $N$-body TreePM code Gadget2 (Springel, 2005). The hydrodynamical equations are solved using a smoothed-particle- 

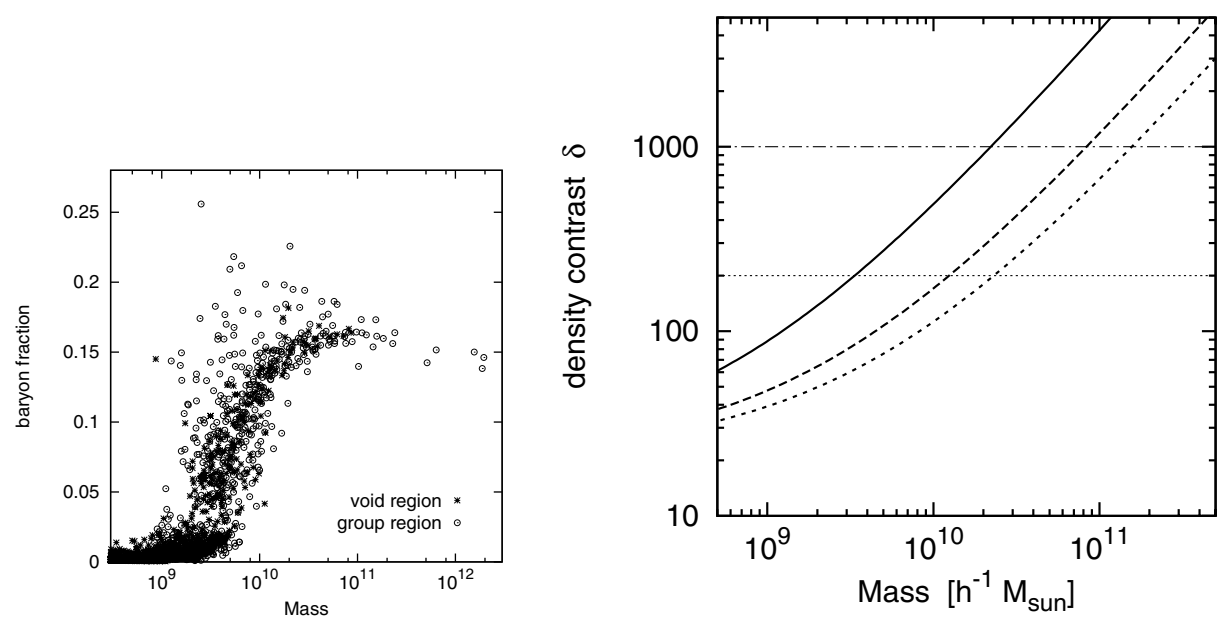

Figure 1. Left panel: Baryon fraction within the virial radius. In a cosmological simulation we studied two distinct regions in detail: a void and a loose group region. Only isolated halos are considered, i. e. substructures are neglected. Right panel: The central gas density contrast as a function of total halo mass for three equation-of-state: $T_{0}=3.8 \times 10^{3} \mathrm{~K}$ (solid line), $8.8 \times 10^{3} \mathrm{~K}$ (dashed lines), and $1.3 \times 10^{4} \mathrm{~K}$ (short-dashed line). Moreover, the density contrast at which cooling becomes important is indicated: for $T_{0}=3.8 \times 10^{3} \mathrm{~K}$ and $8.8 \times 10^{3} \mathrm{~K}$ at $\delta \sim 200$, and for $T_{0}=1.3 \times 10^{3} \mathrm{~K}$ at $\delta \sim 1000$. The crossing between the central density contrast and these horizontal lines give the characteristic mass.

hydrodynamics method based on a entropy-conserving scheme. Radiative cooling is included, assuming a primordial mix of hydrogen and helium. Rates for collisional ionisation, recombination, and cooling are used as given in Katz et al. (1996). The gas is heated by a homogeneous UVB radiation. We have adopted a slightly modified version of the photoionisation and heating rates given in Haardt \& Madau (1996). Star formation and stellar feedback are treated in the code by means of a sub-resolution model in which the gas of the interstellar medium (ISM) is described as a multiphase medium of hot and cold gas. Note, stellar feedback is included in our simulation, but it can only modify the temperature and density of the ISM, we deleberately do not consider galactic winds to focus on the effects of photoheating solely.

Using a multi-mass technique, we simulate a cosmological void region and the environment of a loose, poor galaxy group selected from a computational box of side-lengths $L=50 h^{-1} \mathrm{Mpc}$. To construct suitable initial conditions, we first created a random realization with $2048^{3}$ particles using the $\Lambda$ CDM power spectrum of perturbations of the concordance model $\left(\Omega_{m}=0.3, \Omega_{\Lambda}=0.7, \Omega_{b}=0.04, h=H_{0} /\left(100 \mathrm{~km} \mathrm{~s}^{-1} \mathrm{Mpc}^{-1}\right)=0.7\right.$ and $\left.\sigma_{8}=0.9\right)$. However, later on this very high resolution is only used in the regions of interest. The large-scale gravitational field is represented by more massive particles. The void was simulated with the full resolution, i.e. $2048^{3}$, while the group was simulated with $1024^{3}$.

We compute for each isolated halo in the two regions the baryon fraction within the virial radius, $f_{\mathrm{b}}=\left(M_{\mathrm{star}}+M_{\text {gas }}\right) /\left(M_{\mathrm{star}}+M_{\mathrm{gas}}+M_{\mathrm{dm}}\right)$, see Fig. 1, left panel. One can clearly see that the baryon fraction decreases for masses below $\sim 6 \times 10^{9} h^{-1} M_{\odot}$ (at this mass $f_{\mathrm{b}}$ is half of the average cosmic baryon fraction). Hoeft et al. (2006) showed that this mass scale is very robust even when changing the resolution, or the UV background flux density over a large range of parameters. Fig. 1 also indicates that the characteristic mass is the same for the void and the group region. Moreover, our simulations allow us to study the evolution of the baryon fraction in individual halos. Fig. 2 shows four 


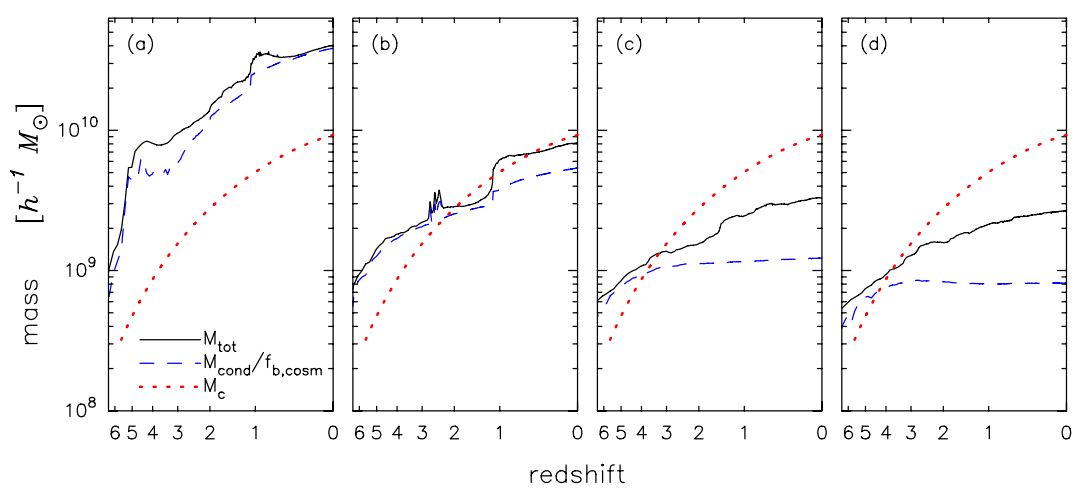

Figure 2. The mass accretion histories for four halos. In each panel the total mass (solid line) and the condensed mass (dashed line) of one halo is shown. Note, the latter is divided by the mean cosmic baryon fraction, $f_{\mathrm{b}, \mathrm{cosm}}$, hence, the naive expectation is that both curves lie on top of each other. This allows to recognise easily where condensation starts to fail, namely when the total mass falls below the characteristic mass, $M_{\mathrm{c}}(z)$, (dotted line).

examples. For more massive halos the evolution of the dark and the baryonic matter runs virtually parallel. In contrast, less massive halos stop to increase their content of condensed baryons, i. e. stars and cold, dense gas, at some redshift. That time coincides very well with the time when the halo mass falls below the characteristic mass, $M_{\mathrm{c}}(z)$.

The evolution of the individual halos shows clearly how photoheating acts on the baryon fraction: The gas is heated in a way, that cooling times in the centre of a halos get longer, hence condensation cannot take place anymore. The time when this happens is not necessarily close to reionisation, instead, depending on the mass and the mass accretion history of a halo, it may also happen close to $z=0$. In the next section we describe a spherical model which allows to derive the characteristic mass analytically.

\section{Why do halos fail to condense gas?}

We wish to derive the mass scale below which halos fail to condense gas in a simple model. As a first step we could adopt the effective equation-of-state found for the low density IGM from numerical simulations. However, we derive this equation explicitly by integrating the thermal evolution of the IGM including atomic hydrogen and helium species, $\{\mathrm{HI}, \mathrm{HII}$, HeI, HeII, HeIII $\}$ and adopting a primordial helium mass fraction, $Y_{\mathrm{p}}=0.24$. All species are assumed to be always in collisional equilibrium. This allows us to follow standard procedures for computing the thermal evolution of the IGM.

Cosmic structures form out of an almost homogeneous matter distribution and so do galaxies. If we wish to follow the thermal history of a small part of IGM we have to know how the density, or alternatively the density contrast $\delta=\rho_{b} / \bar{\rho}_{b}-1$, evolves from the mean density in the beginning to a certain final density. More precisely, we wish to find a function which describes the average density contrast evolution, $\delta\left(t, \delta_{0}\right)$, as a function of the final density contrast, $\delta_{0}$. In Hoeft et al., (in prep) we present a fitting formula for the evolution of the gas density contrast found in the simulations.

The resulting temperature distribution, $T(\delta+1)$, reproduces well the effective equationof-state found in numerical simulations. Fig. 3 shows the resulting temperature distribution for several UVB models. All of them show a power-law for the low density regime, $T=T_{0}(\delta+1)^{\gamma-1}$. For the standard Haardt \& Madau (1996) model we find $T_{0}=3.8 \times 10^{3} \mathrm{~K}$ and $\gamma=1.6 . T_{0}$ increases slightly if we increase only the heat input at high redshift. Our maximal model corresponds to a ten times increased energy per ionising photon for all 

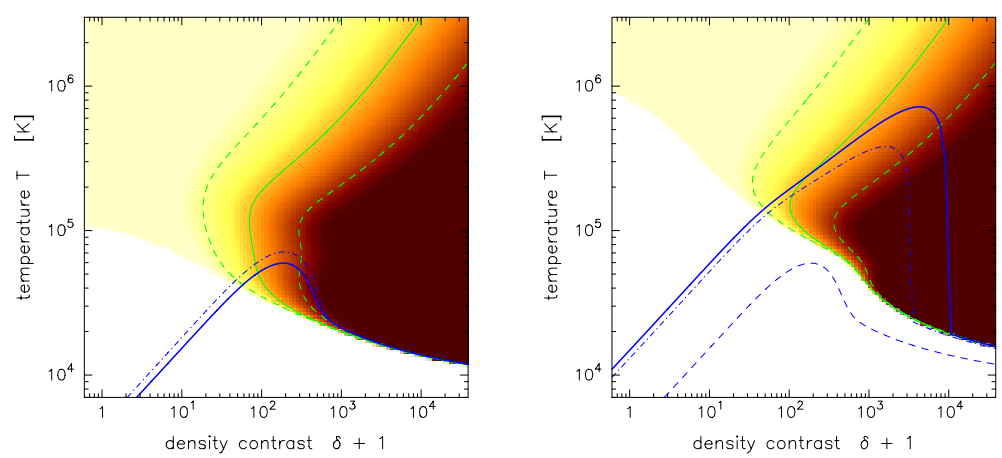

Figure 3. The temperature, $T(\delta+1)$, distribution for several heating models. Left panel: Standard UVB heating model (solid line) and four times increased energy per ionising photon only for $z>2$ (dash-dotted line). One can see the effective equation-of-state at low densities and the thermal balance at high densities. The colour in the background indicates the cooling times. Contours are drawn at 10x (dashed line), $1 \mathrm{x}$ (solid line), and $0.1 \mathrm{x}$ (dashed line) the Hubble time (from the left to the right). Right panel: Beside the standard model (dashed line) also eight (dash-dotted line) and ten (solid line) increased energy per ionising photon is shown. The cooling times are here computed for the last model.

redshifts. For this rather extreme scenario the temperature, $T_{0}$, rises to $1.5 \times 10^{4} \mathrm{~K}$. Beside the effective equation-of-state at low density our integration yields also the transition to thermal balance at higher densities. At even higher densities the temperature lies exactly at the border between heating and cooling, see Fig. 3. We can read out of Fig. 3, right panel at which density contrast the transition to the thermal balance occurs. For the standard heating model this is at $\delta \sim 200$ (maximum of the dashed line). In contrast for UVB models with eight to ten times more energy per ionising photon this shifts to $\delta \gtrsim 1000$ (dash-dotted and solid line, respectively).

Let us return to the question why massive halos can accrete gas and small ones cannot. Since photo heating sets the lowest temperature possible for a given density one can rephrase the questions as follows: Which halos can compress the central gas sufficiently to bring the gas into the regime of thermal balance, i. e. to make cooling times short? To answer this question we compute the gas density profile for a spherically symmetric halo. It is important to notice that with an power-law equation-of-state the gas reaches a finite pressure in the centre of a halo. As we are interested in rather baryon-poor halos a NFW density profile is a reasonable approach for the dark matter halo, which dominates the gravitational force. Fig. 1, right panel shows the central gas density contrast as a function of halo mass. Assuming the standard UVB model for halos of a few times $10^{9} h^{-1} M_{\odot}$ a density is reached where gas enters the realm of thermal balance, i. e. cooling times are short. The mass scale found by integration agrees with that found in the simulation. For more energy per ionising photon a higher halo mass is necessary to reach thermal balance.

\section{More UV heat}

The characteristic mass scale for suppressing gas accretion in dark matter halos is significantly lower than the mass scale of galaxies at which the mass-to-light ratio increases dramatically (v.d. Bosch et al., 2007). Therefore, one can ask what heat input is needed to rise the characteristic mass to a level consistent with observations. We have increased the energy per ionising photon in our heating model by a factor of four and by a factor of eight. In the first case one lowers in general the central density of the halos, see Fig. 1. 
In the second case one needs additionally a higher central density to reach short cooling times, cf. also Fig. 3., right panel. Therefore, the second case represents virtually an upper limit for the heat input into the IGM. This model would lead to a temperature $T_{0} \sim 1.3 \times 10^{4} \mathrm{~K}$ and a characteristic mass above $10^{9} h^{-1} M_{\odot}$.

\section{Conclusions}

We have presented high-resolution simulations including radiative cooling and photo heating. Our simulations allow us to determine robustly the characteristic mass scale below which photo heating reduces the baryon fraction in dwarf galaxy size dark matter halos. Using a Haardt \& Madau (1996) model for the UV background the mass scale amounts to $M_{\mathrm{c}}=6 \times 10^{9} \mathrm{~h}^{-1} M_{\odot}$. The simulations show that halos start to fail accreting gas when the mass of the halo becomes smaller than the characteristic mass $M_{\mathrm{c}}(z)$.

The failure of condensing gas can be traced back to the cooling time in the halo centre, which may exceed the Hubble time. We have set up a spherical model for the gas density profile. Crucial for this model is the effective equation-of-state which we have derived by integrating the thermal history of the IGM using an approximation for the density contrast evolution. This spherical model allows us to derive the characteristic mass for different heating histories of the IGM.

Therefore, the lower mass scale at which galaxy formation fades away may serve as calorimeter for the temperature of the IGM. We find that a heat input with a resulting temperature of $T_{0} \sim 10^{4} \mathrm{~K}$ would be consistent with a characteristic mass scale of few times $10^{10} h^{-1} M_{\odot}$. Note that the temperature quoted above may differ to some extent from that obtained from the Ly $\alpha$ forest since the heat input in the vicinity of even small galaxies might be higher than in those structures probed by the forest.

Finally, the combination of cooling times and the effective equation-of-state shows a remarkable feature, see Fig. 3, right panel: If $T_{0} \gtrsim 1.5 \times 10^{4} \mathrm{~K}$ the equation-of-state runs quasi parallel to the cooling contour lines. Hence, if a galaxy can heat all surrounding gas to this temperatures it will stop to accrete gas for a long time, i.e., radiative feedback may strongly affect the baryon content in a halo.

\section{Acknowledgements}

Numerical simulations has been conducted at the Barcelona Supercomputer Center (BSC) and the CLAMV at Jacobs University, Bremen. This work has been supported by the Deutsche Forschungsgemeinschaft (DFG) under Vo 855/2.

\section{References}

van den Bosch, F. C., Yang, X., Mo, et al. MNRAS 376, 841

Haardt, F. \& Madau, P. 1996, ApJ 461, 20

Hoeft, M., Yepes, G., Gottlöber, S. \& Springel, V. 2006, MNRAS 371, 401

Katz, N., Weinberg, D. H. \& Hernquist, L. 1996, ApJS 105, 19

Springel, V. 2005, MNRAS 364, 1105 